\title{
COVID-19 la pandemia que nos prepara para un nuevo enfoque de salud-enfermedad
}

Tras el primer brote de COVID-19 reportado en Wuhan, Provincia de Hubei, China en diciembre de 2019(1), la Organización Mundial de la Salud (OMS) alertó sobre la emergencia sanitaria global el 11 de marzo de 2020(2) lo que obligó a los gobiernos a adoptar medidas inmediatas y establecer acciones de manejo coordinadas; desde entonces, el mundo se enfrenta a una situación comparable con la causada por la Gripe española de 1918.

La pandemia por COVID-19 ha marcado cambios para la humanidad, los países más afectados han impuesto importantes restricciones a la movilidad, necesarias para cortar con la cadena de transmisión de toda infección respiratoria, pero que se tornan difíciles de cumplir por largo tiempo. La adopción de medidas sanitarias básicas como lavado de manos frecuente (con agua y jabón) o el uso de alcohol desinfectante, el uso de tapabocas en todo espacio público, mantener una distancia social $(2 \mathrm{~m})$, restricción de encuentros y reuniones, trabajo de oficina en casa, limitado acceso a educación presencial en todos los niveles y monitoreo de contactos con inteligencia artificial han sido las más discutidas, pero también las más efectivas.

Estas medidas, han generado desconcierto y descontento en muchas personas, en razón de que no surgieron desde el día cero de la pandemia. Sin embargo, para que los tomadores de decisiones, científicos, salubristas, epidemiólogos puedan generar recomendaciones y los entes de decisión puedan implementarlas fue necesario primero entender el comportamiento del nuevo virus SARS-CoV-2 causante de la infección COVID-19 en las poblaciones, y por ello, la adopción de una, otra o la combinación de ellas, se definió de forma específica en cada país e incluso en cada región dentro de un país.

Analicemos algunos aspectos del manejo de la pandemia por COVID-19:

Respecto a las restricciones de movilidad; en países como Colombia y otros de bajos y medios ingresos, restringir la movilidad de sus habitantes supuso entre otros efectos desempleo y hambre. La tasa de desempleo en Colombia en la pre-pandemia, estaba en 9,7\% en 2019(3), alcanzando un máximo de $22 \%$ en mayo y un $15,8 \%$ reportado en septiembre de 2020 según cifras del Sistema Estadístico Nacional (SEN)(4). Frente a esta realidad y después de seis meses de confinamiento, los efectos negativos en la economía de los trabajadores informales y ciertos sectores (arte, cultura, deporte, recreación) no se hicieron esperar.

La restricción en la movilidad también agudizó problemas sociales de violencia intrafamiliar y salud mental (ansiedad y depresión). Un estudio realizado en Estados Unidos con 69 millones de personas señala a los problemas siquiátricos como un factor de riesgo entre las secuelas que deja COVID-19 para aquellos sobrevivientes a la infección, para un mejor entendimiento de tales secuelas, tendrán que dirigirse estudios de cohorte en poblaciones específicas y se tendrá que analizar en los próximos meses y años la magnitud de estos efectos desde el punto de vista social(5).

En contraste, como efectos positivos de las restricciones de movilidad uno de los más evidentes fue una mejor calidad del aire, solo en marzo reportes de Naciones Unidas afirman que la disminución de la actividad económica-industrial en China redujo $25 \%$ de la emisión de $\mathrm{CO}_{2}$ durante cuatro semanas ${ }^{(6)}$. Los efectos positivos sobre los ecosistemas, la biodiversidad y los recursos naturales en los años siguientes se deberán reforzar no solo para hacer un planeta más sostenible, sino también porque la aparición de nuevas infecciones, tales como la causada por SARS-CoV-2 está estrechamente vinculada con nuestro efecto antropogénico; prácticas como el uso de especies silvestres con fines alimentarios (pangolines, murciélagos, palomas, pájaros, roedores, visones, felinos, caninos) se deberán suprimir, si como especie humana "Homo sapiens sapiens" nos queremos mantener; tendremos que asumir un rol más activo en el cuidado, protección, uso y consumo de recursos naturales. Estudios con enfoque de una sola salud (One-Health) deberán ser conducidos para identificar los riesgos de aparición de enfermedades infecciosas nuevas y emergentes, las cuales, resultan del desequilibrio sostenido en la interacción humano-animal-ambiente. 
Por otro lado, en relación con las medidas sanitarias; en países y regiones con baja infraestructura sanitaria o con prácticas de defecación abierta tales como Sudeste de Asia o África, países de Centro América, o en Sur América (Brasil, Ecuador, Perú, Bolivia, Colombia), cumplir con tales medidas sanitarias básicas supuso limitaciones para sus habitantes y, por tanto, el control de SARS-CoV-2 en numerosas regiones resultó deficiente. En contraste, la adopción de medidas sanitarias podría ser favorable en el futuro cercano, no solo para el control de esta y otras infecciones respiratorias (gripa, influenza, tuberculosis), sino también, para la reducción de ambientes y reservorios de infecciones gastrointestinales, infecciones transmitidas por vectores (dengue, zika, chikunguña, chagas) y problemas relacionados con malas prácticas sanitarias tales como la desnutrición infantil. Estudios en este campo deberán ser orientados.

Otro aspecto de análisis son las limitaciones de acceso a la educación presencial; en este sentido, los niños han sido severamente afectados, aunque no por razones lúdicas simplemente, sino porque quienes dependían del acceso a los programas de alimentación escolar, cuyos padres o cuidadores perdieron sus empleos durante la pandemia, tienen alto riesgo de sufrir retraso en el crecimiento y desarrollo. Según el Reporte Global de Nutrición ${ }^{(7)}$ en Colombia antes de la pandemia alrededor de 3 millones de personas se encontraban en riesgo de hambre, y se prevé que esta cifra se triplique en la pos-pandemia. Se deberá entonces, garantizar y fortalecer la protección infantil en procura de generaciones de niños en la pos-pandemia con el menor efecto posible en su salud nutricional, mental y emocional.

Por su parte, el uso de inteligencia artificial en el monitoreo de contactos ha supuesto toda clase de imaginarios entre las personas al sentir vulnerada su privacidad. En China, por ejemplo, la instalación de aplicaciones $(A p p)$ para monitorear el desplazamiento y los contactos cercanos de pacientes diagnosticados con COVID-19 durante los meses de confinamiento y posteriores no fue optativa. Otros países han optado por la no imposición y han limitado su uso para contextos específicos como aeropuertos para viajeros internacionales. En este aspecto es importante señalar que el monitoreo con geo-posicionadores (GPS) es una práctica rutinaria en todo el mundo desde la invención del "smartphone" en 1992 y de todos quienes hacemos uso de redes sociales. La pregunta que vale la pena hacer es, hasta donde la pandemia por COVID-19 está imponiendo nuevas restricciones, cortando libertades o vulnerando la privacidad y hasta donde las sociedades han reaccionado por desconocimiento y se han tardado en asumir la responsabilidad individual y colectiva para el control de esta pandemia.

Pese a todo lo anterior, por primera vez los ciudadanos se han visto en la necesidad de familiarizarse con los principios básicos de epidemiología y salud pública, han seguido con interés las cifras de casos nuevos (incidencia), número de fallecidos (mortalidad) e incluso las tasas y cifras por millón de habitantes y los comparativos entre países. Ahora el trabajo lo debe realizar cada individuo, analizar esa información y adoptar un comportamiento responsable debe estar por encima de un decreto o una normatividad, debe ser parte del sentido común frente a esta pandemia y a futuras epidemias que eventualmente ocurrirán.

Finalmente, con el nuevo SARS-CoV-2 y el desarrollo de medicamentos y vacunas ha sido un tema de amplia discusión. Al respecto, la inmunización de las poblaciones, entendida como el entrenamiento y preparación de las defensas naturales del organismo para detectar y combatir contra microorganismos, de manera que si un organismo se expone a patógenos podrá destruirlos evitando enfermar. La inmunización en las poblaciones se puede lograr de dos maneras principales: (i) Por exposición al agente patógeno repetidamente (Inmunización de rebaño) o (ii) Con la aplicación de vacuna (Inmunización sintética).

En la inmunización de rebaño, estrategia adoptada durante la pandemia por COVID-19 por países como Suecia, supone acudir a la voluntad de los habitantes de mantener aislamiento y adoptar medidas sanitarias hasta lograr que un $70 \%$ de ello se infecten y puedan convivir con el virus, ya inmunizados. Esta estrategia, sin embargo, plantea cuestiones éticas importantes, pues para lograr ese porcentaje de individuos inmunizados un alto número de personas deberá morir. El caso Suecia, país escandinavo con 9.84 millones de habitantes, que optó por la inmunidad de rebaño, registró durante la primera ola de la pandemia una tasa de mortalidad de 56.98 per cápita, considerada la más alta en Europa, con un total de muertos por coronavirus de 51.405 hasta finales de junio 2020. Sin embargo, en la segunda ola a principios de noviembre se ubica como una de las tasas más bajas en la región( ${ }^{(8)}$. Aquí, surgen cuestiones sobre si esta estrategia funciona para países con un producto 
interno bruto (PIB) alto (55.856 US\$ per cápita)(9), un eficiente sistema de salud y una inversión social como el de Suecia, donde sus habitantes pueden optar por el aislamiento sin poner en riesgo su nutrición o supervivencia, o si aún es temprano para sacar conclusiones. En todo caso, para considerar exitosa una u otra alternativa se deberá analizar otros factores como la frecuencia de exposición y reinfección del individuo, su capacidad inmunológica, su genética, factores sociales, culturales, ambientales diversos en los países.

Respecto a la segunda estrategia de inmunización basada en vacunas, se están desarrollando 169 vacunas candidatas contra COVID-19, de ellas, 26 se encuentran en ensayos en humanos(10). Entre las estrategias más prometedoras utilizadas por los desarrolladores de vacunas se tienen las basadas en virus inactivados, ácido desoxirobonucleico (ADN) recombinante, ácido Ribonucleico (ARN), ácido Ribonucleico mensajero (mARN), lisados de partículas virales (VLP), viriones inactivados, vectores virales no replicativos, vectores de adenovirus tipo $5,26,5 s+26 s$, vectores de replicación viral, subunidades proteicas empaquetadas con adyuvantes; cada una con un mecanismo de acción, seguridad y efectividad particular.

En el top de los países que compiten por el diseño, aprobación y comercialización de la primera vacuna contra SARS-CoV-2 se encuentran China (Sinovac; Sinopharm; CanSino Biological Inc,), India (Bharat Biotech), Reino Unido (Oxford-Astrazeneca; Janssen Pharmaceutical Companies-UK), Rusia (The Gamaleya-Sputnik V), Estados Unidos (Novavax, Moderna), Alemania (BioNTech/Fosun Pharma/Pfizer)(11). De ellos, los resultados preliminares sobre efectividad y seguridad más promisorios reportados hasta ahora son las vacunas de Moderna con 94,5\% efectividad de su candidato mRNA-1273 asegurando que podría prevenir casos severos de COVID-19.

Seguidamente el consorcio BioNTech/Fosun Pharma/Pfizer con su candidato BNT162b2 también basada en ARN, anunció un 90\% de efectividad para prevenir COVID-19 en pacientes sin infección previa y la aprobación para la producción inicial de 50 millones de dosis en los últimos dos meses de 2020.

La tercera en la lista es la vacuna de Rusia, Sputnik $V$, la cual se basa en inmovilización de la proteína $S$-spike de SARS-CoV-2 en un vector adenoviral ( $r A d 26$ and $r A d 5$ ), cuya efectividad actual es del 92\% y para la cual, once países de Asia, India y Sur América han aportado voluntarios para evaluar la seguridad hasta ahora garantizada.

En esta carrera científica para perseguir la inmunización contra COVID-19 mediante vacunación, los retos que se tienen ahora son disminuir todos los riesgos de su aplicación, a día de hoy, de los tres candidatos, Moderna y BioNtech-Pfizer han reportado enrojecimiento y mínimo dolor, y Galerma ha reportado dolor de cabeza leve; sin embargo, serán necesarios estudios de seguimiento a largo plazo para garantizar la efectividad y seguridad necesarios. Así también, se tendrá que garantizar el acceso equitativo a la vacuna, asegurar una eficiente cadena de suministro y se tendrán que priorizar los grupos de riesgo en la estrategia de vacunación. La aceptación de la vacuna en la población, deberá también competir con la desinformación, los grupos negacionistas, los movimientos antivacunas, los conflictos geo-políticos y económicos de los países.

Sin embargo, no es la primera vez que el mundo se enfrenta al uso de vacunas, es más, hoy en día numerosos países tropicales exigen portar carnet de vacunación a sus viajeros, para gripe, fiebre amarilla, BCG (antituberculosis), DPT (Difteria, tosferina, tétanos). Con la vacunación se salvan alrededor de 3 millones de vidas por año. Garantizar la vacunación no es un reto exclusivo de la ciencia, se requiere además voluntad de los gobernantes, compromiso y ética de las compañías fabricantes, así como voluntad y compromiso de las personas que permitan transitar por esta situación sanitaria global con los mínimos efectos negativos posibles.

\section{Milena Guerrero F. Grupo de Investigación Salud Pública}

\section{Referencias}

1. Na Z, Dingyu Z, Wang W, Xiangwang L, Bo Y, Jingdong S, et al. (2020). A Novel Coronavirus from Patients with Pneumonia in China, 2019. The New England Journal of Medicine. https://doi.org/10.1056/NEJMoa2001017

2. Organización Mundial de la Salud (2020). COVID-19. Cronología de la actuación de la OMS. Retrieved from: https://www.who.int/es/news/item/27-04-2020-who-timeline---covid-19 
3. World Bank. (2020). International Labour Organization, ILOSTAT database. Data retrieved in June 21, 2020 from: https://data.worldbank.org/indicator/SL.UEM.TOTL.ZS?locations=CO

4. DANE (2020). Gran encuesta integrada de hogares (GEIGH) mercado laboral. Retrieved in November 19 of 2020 from: https://www.dane.gov.co/index.php/estadisticas-por-tema/mercado-laboral/empleo-y-desempleo

5. Maxime T, Sierra L, Geddes JR, Harrison PJ. Bidirectional associations between COVID-19 and psychiatric disorder: retrospective cohort studies of 62354 COVID-19 cases in the USA. (2020). The Lancet Psychiatry. DOI: https://doi.org/10.1016/S22150366(20)30462-4

6. United Nations. (2020). La cuarentena por el coronavirus mejora la calidad del aire, pero no sustituye la acción climática. Retrieved November 9 of 2020 from: https://news.un.org/es/story/2020/03/1471562

7. GNR. (2020). Global Nutrition Report: Action on equity to end malnutrition. Bristol, BS16FL, UK.

8. SCB Statistics Sweden's website. (2020). Excess mortality in Sweden is followed by mortality deficit. Retrieved November 9 of 2020 from: https://www.scb.se/en/About-us/news-and-press-releases/excess-mortality-in-sweden-is-followed-by-mortality-deficit/

9. OECD Organización para la cooperación económica y el desarrollo (2020). Retrieved November 19 of 2020 from: https://data.oecd.org/sweden.htm

10. WHO World Health Organization. (2020). La carrera por una vacuna contra la COVID-19. Retrieved November 19, 2020 from: https://www.who.int/es/emergencies/diseases/novel-coronavirus-2019/covid-19-vaccines

11. WHO World Health Organization. (2020). Draft landscape of COVID-19 candidate vaccines. Retrieved November 19 of 2020 from: https://www.who.int/publications/m/item/draft-landscape-of-covid-19-candidate-vaccines 